\title{
Recuperação Hidrofóbica de Polipropileno Tratado por VUV ou Plasma
}

\author{
Cesar H. Wanke, Luiz G. Barbosa \\ Programa de Pós-Graduação em Ciência dos Materiais, UFRGS
}

Júlia V. M. Hübner

Departamento de Engenharia Química, UFRGS

\author{
Flavio Horowitz \\ Departamento de Física, UFRGS
}

Raquel S. Mauler, Ricardo V. B. de Oliveira
Departamento de Química Orgânica, UFRGS

Resumo: Tratamentos superficiais por plasma e ultravioleta de vácuo (VUV) foram utilizados para introduzir grupos funcionais contendo oxigênio em amostras de polipropileno, com objetivo de modificar sua molhabilidade. Análises por ATR-FTIR, AFM e ângulo de contato (AC) foram utilizadas para analisar as mudanças químicas e físicas na superfície do PP em função do tempo. Os resultados mostraram que as mudanças na molhabilidade das amostras tratadas por VUV ocorreram principalmente devido à alteração química da superfície. Nas amostras tratadas por plasma, o envelhecimento ocorreu mais rapidamente que as amostras tratadas por VUV. Para ambos os tratamentos, provavelmente ocorreu um rearranjo das cadeias durante o envelhecimento, além de uma possível reticulação da superfície na amostra tratada por VUV.

Palavras-chave: Polipropileno, ultravioleta, plasma, envelhecimento.

\section{Hydrophobic Regeneration of Polypropylene with VUV and Plasma Treatments}

\begin{abstract}
Surface treatments using plasma and vacuum ultraviolet radiation (VUV) were used to introduce oxygen-containing functional groups in samples of polypropylene, in order to modify its wettability. ATR-FTIR, AFM and contact angle (CA) measurements were used to analyze the chemical and physical changes on the PP surface as a function of time. The results showed changes in wettability of the VUV-treated samples mainly due to chemical modification of the surfaces. For plasma-treated samples, aging occurred faster than for the VUV-treated ones. For both treatments, a rearrangement of the polymer chains during aging has probably occurred, with possible crosslinking of surface macromolecules for the VUV-treated samples.
\end{abstract}

Keywords: Polypropylene, UV, plasma, aging.

\section{Introdução}

Materiais a base de polipropileno são usados grandemente na indústria automotiva ${ }^{[1]}$ e em embalagens ${ }^{[2]}$. Por ser um polímero hidrofóbico, o polipropileno possui baixa molhabilidade e consequentemente baixa adesão; portanto, para muitas das aplicações do PP é necessário aumentar a molhabilidade do polímero. Vários métodos estão disponíveis para modificar superfícies de polímeros, como corona ${ }^{[3-5]}$, fotoquímica envolvendo a exposição da superfície à radiação ultravioleta de vácuo, VUV $(200 \mathrm{~nm}<\lambda<100 \mathrm{~nm})^{[6-13]}$ e o tratamento por plasma ${ }^{[14-18]}$. A molhabilidade como uma propriedade de superfície, pode ser ajustada tanto por modificações na energia livre quanto topográficas ${ }^{[19]}$.

As principais mudanças na superfície dos polímeros quando estes estão sujeitos ao tratamento por plasma e/ou radiação (UV e VUV), são quebra da cadeia principal, reticulação e formação de produtos voláteis. A diferença está em como isso ocorre e quais são as espécies responsáveis por essas mudanças. Enquanto que no tratamento por plasma o principal agente é o bombardeamento pelas espécies ativas do plasma (íons, elétrons), no tratamento por radiação ultravioleta, os fótons são os principais responsáveis pelas mudanças superficiais ${ }^{[20]}$.

Com a crescente utilização desses polímeros modificados uma questão se faz importante: como a modificação se comporta no tempo? O envelhecimento da superfície do polímero modificado é caracterizado pelo retorno da hidrofobicidade com o decorrer do tempo, ou seja, ao estado de molhabilidade anterior ao tratamento. Embora existam muitos estudos de envelhecimento de polímeros tratados por plasma ${ }^{[16,21-23]}$, os polímeros tratados por VUV têm sido negligenciados neste contexto ${ }^{[24]}$. Um fator importante na análise da recuperação hidrofóbica é o próprio tratamento, pois dependendo das condições e do tipo de tratamento a recuperação hidrofóbica pode ser mais ou menos lenta ${ }^{[16,25,26]}$.

Neste trabalho amostras de polipropileno foram tratadas por VUV e por plasma, sendo submetidas ao envelhecimento. As modificações químicas e físicas das superfíes do PP em função do tempo de armazenagem foram analisadas por Espectroscopia de Infravermelho com Transforma de Fourier no modo Refletância

Autor para correspondência: Ricardo V. B. de Oliveira, Química Orgânica, Instituto de Química, Universidade Federal do Rio Grande do Sul - UFRGS, Bento Gonçalves, 9500, Agronomia, CEP 91501-970, Porto Alegre, RS, Brasil, e-mail: ricardo.oliveira@iq.ufrgs.br 
Total Atenuada (ATR-FTIR), Microscopia de Força Atômica (AFM) e medidas de Ângulo de Contato estático (AC).

\section{Experimental}

\section{Materiais}

As amostras de polipropileno (3,5 g/10 min, Braskem S/A) utilizadas nesse trabalho foram moldadas por injeção na forma de chapas com medidas $2 \times 25 \times 25 \mathrm{~mm}^{3}$. Antes de serem utilizadas, as amostras foram limpas por ultrassonicação em banho de acetona (grau analítico, Vetec) por 30 minutos, secas ao ar e armazenadas em dessecador.

\section{Tratamento por VUV}

Neste trabalho utilizou-se uma lâmpada de Deutério $(\lambda=165 \mathrm{~nm}$, $140 \mathrm{~W})$. Inicialmente as amostras foram irradiadas em atmosfera de nitrogênio (câmara preenchida) durante 15 minutos para ativação da superfície. Após este tempo desligou-se a lâmpada e preencheu-se a câmara com oxigênio para que ocorressem as reações com a superfície ativada e as amostras fossem funcionalizadas. Elas ficaram imersas em oxigênio durante tempos de 10 a 60 minutos (tempos de tratamento). Todas as amostras foram posicionadas a uma distância fixa de 26,6 mm (distância de máxima intensidade) da janela. O equipamento utilizado encontra-se no Laboratório de Fotoquímica e Superfícies (IQ/UFRGS), sendo que o esquema ilustrativo e a fotografia de sua montagem são mostrados na Figura 1. Além disto, é possível notar que vários gases ou misturas de gases podem ser utilizados $\left(\mathrm{N}_{2}\right.$ e $\mathrm{O}_{2}$ no nosso caso) e a distância amostra-janela da lâmpada pode ser variada. Neste trabalho, as amostras modificadas por esse processo serão identificas por amostras tratadas por VUV.

\section{Tratamento por plasma}

Neste tratamento, as amostras foram expostas ao plasma de $\mathrm{O}_{2}$ durante 6 a 40 minutos (tempo de tratamento) em descarga de radiofrequência, com uma potência de $50 \mathrm{~W}$ e pressão de trabalho de 5,1 Pa (conhecido como Dry Plasma Etching). Mais detalhes sobre a influência dos parâmetros de tratamento e os resultados obtidos podem ser consultados no trabalho publicado anteriormente ${ }^{[27]}$. As amostras modificadas por esse processo serão identificas no decorrer deste trabalho como amostras tratadas por plasma.

\section{Envelhecimento}

Para a avaliação do envelhecimento, foram escolhidas as amostras com tempos de tratamento por VUV de 20 minutos (V20) e de tratamento por plasma de 20 minutos (P20) e demais condições como especificado acima. As amostras tratadas foram avaliadas nos tempos de envelhecimento de $0,3,7,14,21$ e 28 dias, sendo armazenadas em dessecador à temperatura e pressão ambientes até o dia de análise.

\section{Caracterização das amostras}

Para avaliação das mudanças químicas na superfície, as amostras foram analisadas por Espectroscopia de Infravermelho com Transformada de Fourier (FTIR) utilizando-se um espectrofotômetro Alpha-E da Brucker (cristal de ZnSe) no modo ATR (refletância total atenuada), localizado no Lab. Multiusuário de Análise Térmica (LAMAT - IQ/UFRGS). Cada espectro foi adquirido com 32 scans numa resolução de $4 \mathrm{~cm}^{-1}$ na faixa de $600 \mathrm{a} 4000 \mathrm{~cm}^{-1}$.

Com o objetivo de verificar mudanças na topografia e determinar a rugosidade das amostras, foram realizadas análises por Microscopia de Força Atômica (AFM). Neste trabalho foi utilizado o microscópio SPM Agilent modelo 5500. As medidas de rugosidade (Rrms) foram feitas de pelo menos duas imagens com $5 \times 5 \mu \mathrm{m}^{2}$, a fim de se diminuir a incerteza dos valores obtidos.

Medidas estáticas do ângulo de contato foram feitas em temperatura ambiente com água destilada. Neste trabalho foi utilizado o método Gota Séssil, sendo o valor do ângulo de contato calculado usando o software SurftensTM. Cinco gotas foram consideradas e cada gota foi medida pelo menos duas vezes. Os valores de ângulo de contato foram calculados pela média aritmética dos valores desprezando-se os valores máximos e mínimos. O equipamento utilizado é de construção própria e encontra-se no Laboratório de Laser e Óptica (IF/UFRGS).

Todas as análises foram feitas entre dois e cinco minutos após o tratamento.

\section{Resultados e Discussões}

A Figura 2 mostra os espectros ATR-FTIR das amostras não tratada (PP) e tratadas por VUV nos tempo de 10, 20, 30, 40,

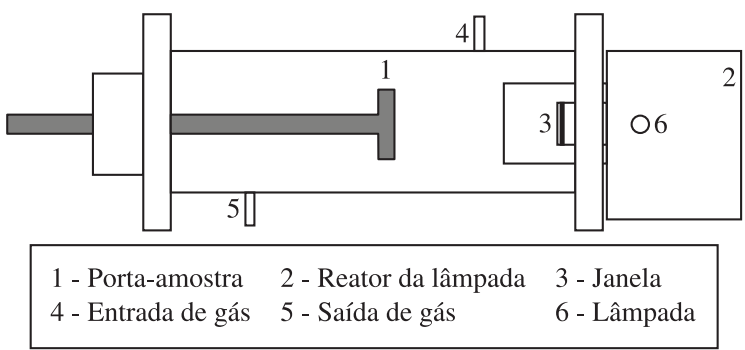

(a)

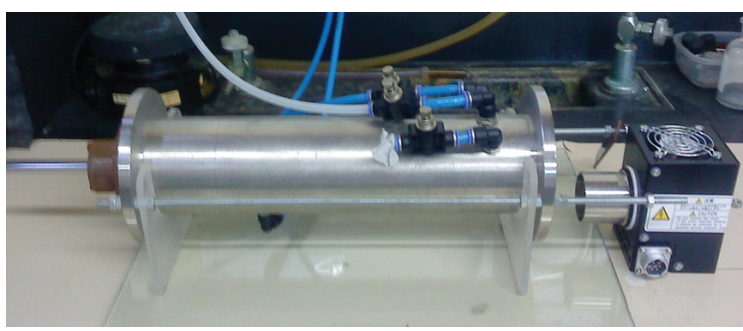

(b)

Figura 1. a) Figura esquemática; e b) fotografia da câmara utilizada para o tratamento com VUV.

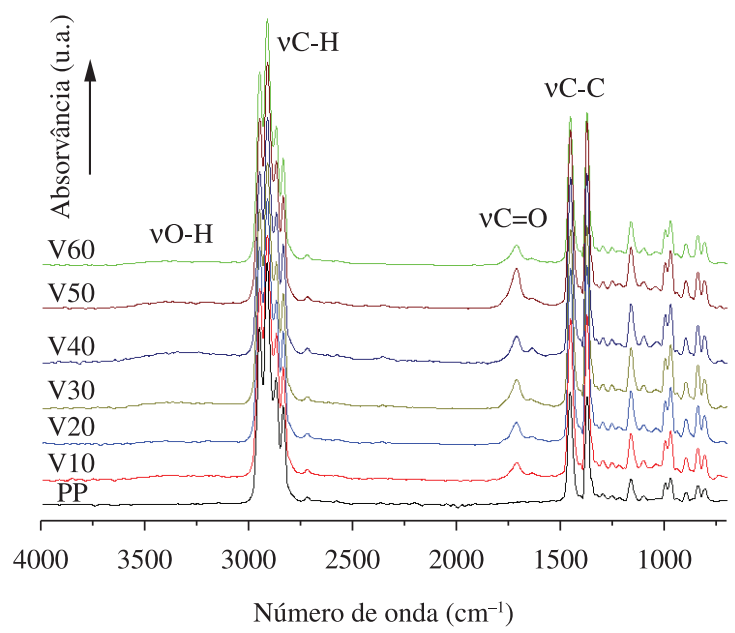

Figura 2. Espectros ATR-FTIR do PP não tratado e das amostras tratadas por VUV. 
50 e 60 minutos (V10 a V60, respectivamente). Os espectros indicam a formação de grupos funcionais, mudança observada no aparecimento de bandas que não existiam no PP sem tratamento. Estas bandas estão localizadas nos espectros em torno de 3200,

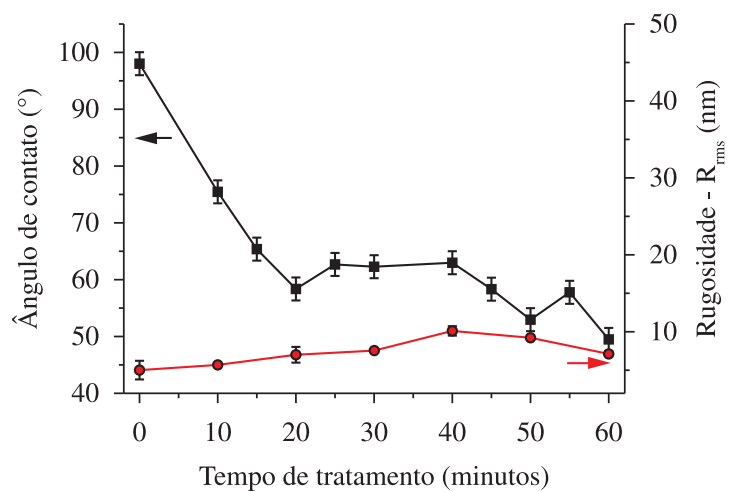

Figura 3. Ângulo de Contato e Rugosidade em função do tempo de funcionalização para as amostras tratadas por VUV.

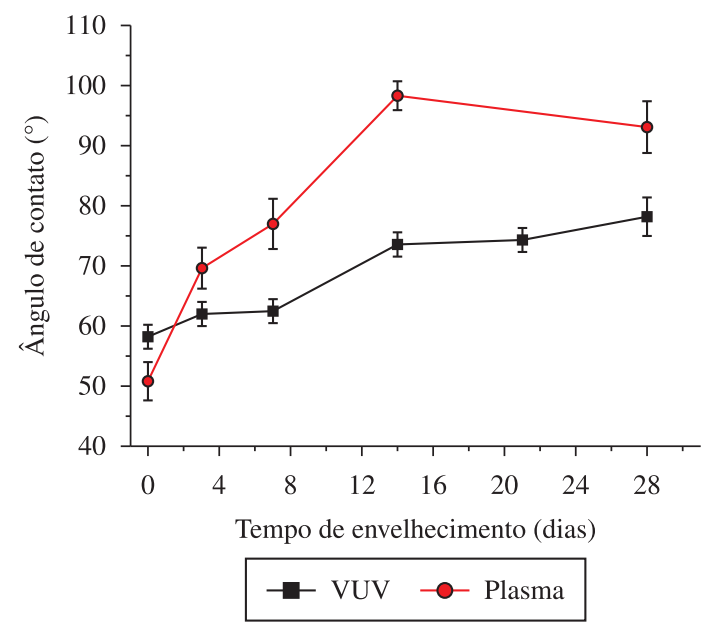

Figura 4. Influência do tempo de envelhecimento no valor do ângulo de contato das amostras tratadas por VUV e Plasma.

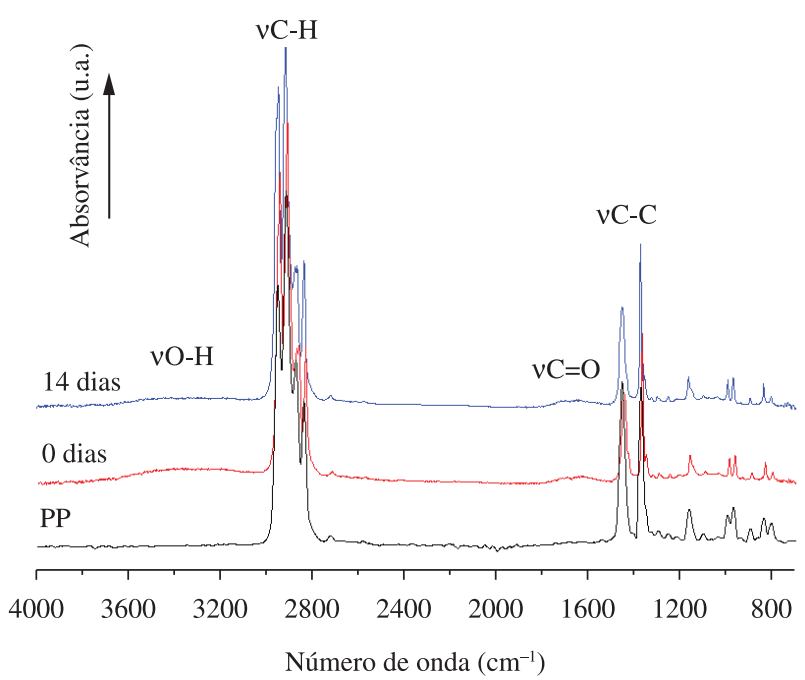

(a)
1720, $1644 \mathrm{~cm}^{-1}$, correspondendo respectivamente ao estiramento da hidroxila $[\mathrm{vO}-\mathrm{H}]$ e carbonila $[\mathrm{vC}=\mathrm{O}](\text { forte e fraco })^{[28]}$.

A Figura 3 mostra a influência do tempo de tratamento por VUV no valor do ângulo de contato (AC) e na rugosidade (Rrms) das amostras. Antes do tratamento, o ângulo de contato do PP é igual a $98^{\circ}$. Por uma análise geral dos valores de ângulo de contato, pode-se considerar que o AC diminui consideravelmente com o tempo até $58^{\circ}$ (20 minutos), permanece aproximadamente constante para tempos entre 25 e 40 minutos, quando se observa novamente uma queda.

Quanto à rugosidade, a Figura 3 mostra que seu valor praticamente não mudou, indo de 5,0 nm (PP não tratado) até cerca de 7,0 $\mathrm{nm}$ (60 minutos). Levando-se em conta o índice de carbonila, que variou de $1 \%$ (PP não tratado) para 15\% (30 minutos) e depois permaneceu aproximadamente neste valor para tempos posteriores, observa-se que a mudança na molhabilidade é devido principalmente à mudança química da superfície. No caso das amostras tratadas por plasma, o ângulo de contato ficou em torno de $50^{\circ}$, embora tenha atingido um mínimo de $24^{\circ}$ após 15 minutos de tratamento. Já a rugosidade aumentou para cerca de $45 \mathrm{~nm}$ após 15 minutos e permaneceu aproximadamente neste valor para tempos posteriores, conforme pode ser visto em mais detalhes no artigo publicado anteriormente ${ }^{[21]}$.

Sabendo-se que a molhabilidade tende, após certo tempo, ao valor de antes da modificação da superfície, efeito chamado envelhecimento ou recuperação hidrofóbica, foram medidos os valores do ângulo de contato nos tempos de $0,3,7,14,21$ e 28 dias de envelhecimento. A Figura 4 mostra a evolução do valor do AC como função do tempo de envelhecimento. Após o tratamento, correspondente ao dia zero no envelhecimento, o valor do AC é cerca de $51^{\circ}$ para a amostra tratada por plasma (P20) e $58^{\circ}$ para a amostra tratada por VUV (V20). Comparando-se as curvas, pode-se observar que a amostra tratada por plasma envelhece mais rapidamente que a amostra tratada por VUV. Após 7 dias de envelhecimento, o valor do $\mathrm{AC}$ da amostra tratada por plasma aumenta cerca de $30^{\circ}$, enquanto que o valor da tratada por VUV aumenta apenas cerca de $4^{\circ}$.

Segundo trabalhos publicados por outros pesquisadores, existem várias explicações possíveis para o envelhecimento de superfícies poliméricas modificadas. Entre tais, pode-se citar a migração de espécies da superfície para o interior (bulk $)^{[21,29]}$, rearranjo do polímero modificado ${ }^{[21,30,31]}$ e reação com a atmosfera

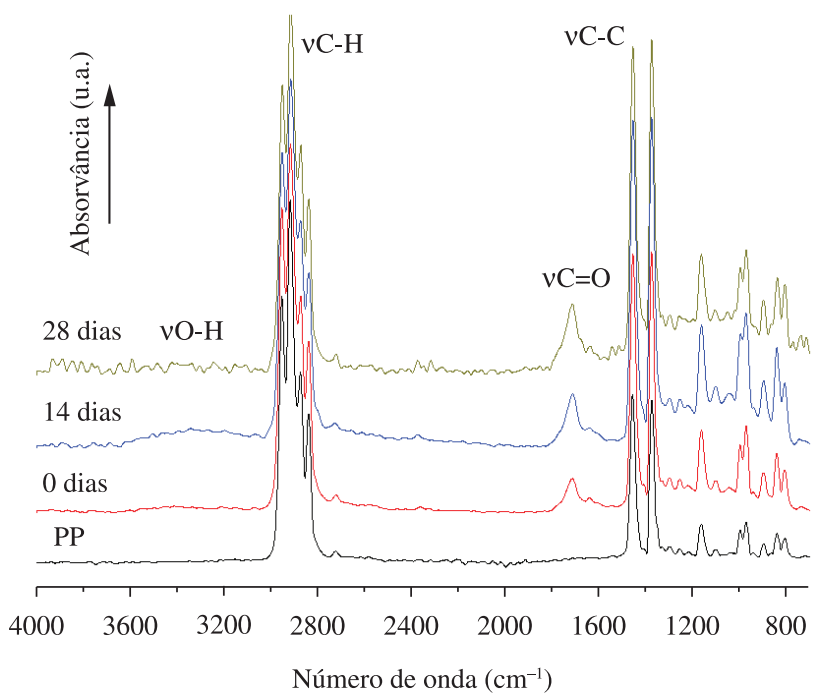

(b)

Figura 5. Espectros ATR-FTIR das amostras envelhecidas que foram tratadas por a) plasma e; b) VUV. 

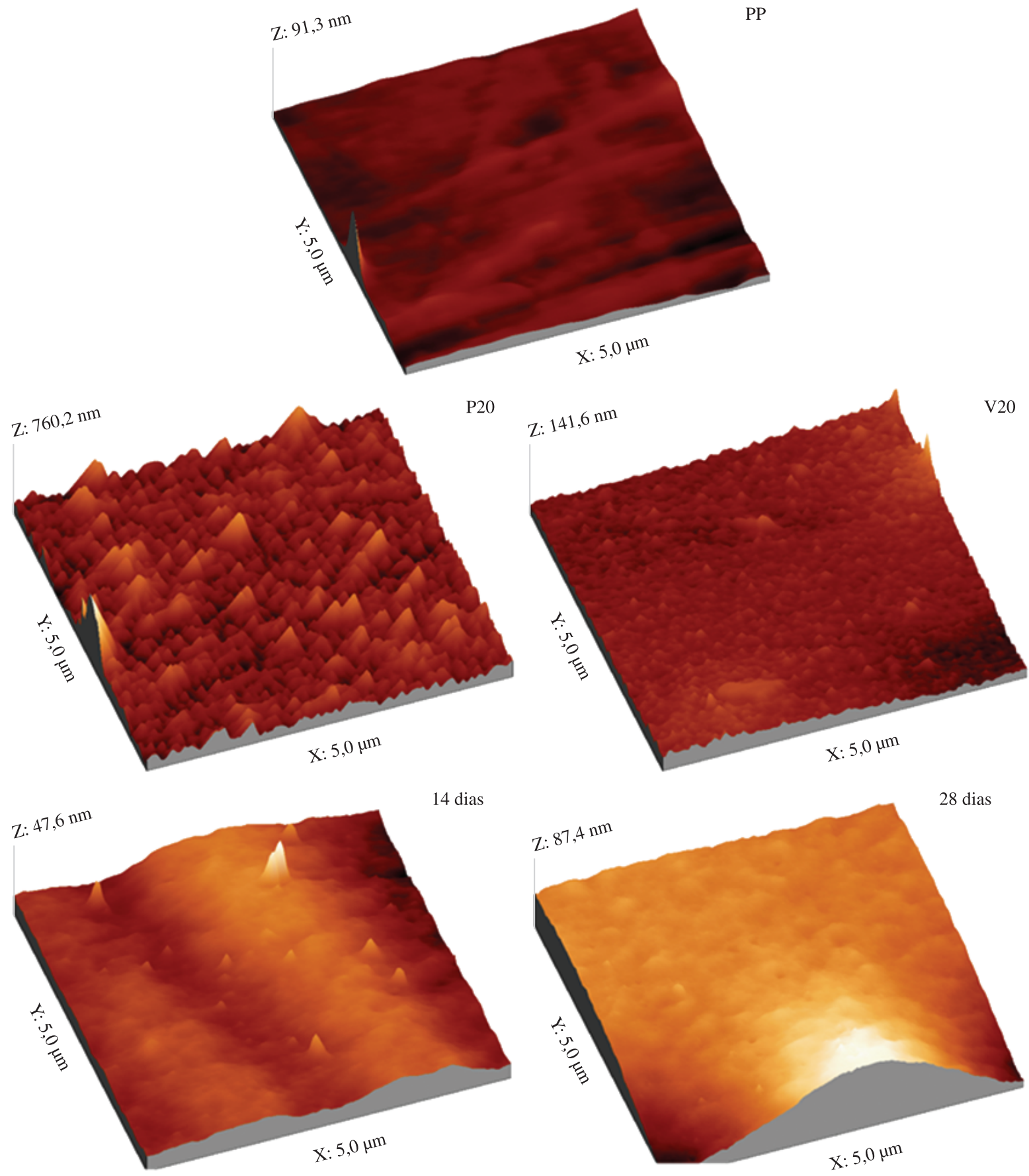

Figura 6. Representações tridimensionais obtidas por microscopia de força atômica para as superfícies do PP não tratado, das amostras tratadas por 20 minutos em plasma (P20) e VUV (V20) logo após o tratamento e para as mesmas amostras envelhecidas por 14 e 28 dias, respectivamente.

de espécies ativas aprisionadas que foram introduzidas na superfície durante o tratamento ${ }^{[32]}$. Além dessas, tem a hipótese apresentada por Yasuda et al. ${ }^{[33]}$ que consideram que, enquanto as cadeias nas camadas mais externa da superfície se acomodam por efeito termodinâmico, os grupos funcionais são ocultados. Ou seja, a quantidade de tais grupos na superfície tende a diminuir. Em geral, acredita-se que dois ou todos estes efeitos influenciam no envelhecimento das amostras modificadas.

As cadeias da região mais externa da superfície dos polímeros possuem grau de liberdade maior que as da região mais interna.
Durante o tratamento por plasma as partículas deste transferem energia para a superfície do polímero de modo a causar mudanças físicas e químicas. Após o tratamento, as cadeias da região mais interna tendem a se acomodar, por razões termodinâmicas, fazendo com que haja um rearranjo destas cadeias. Isso implica numa alteração na topografia e induziria a migração dos grupos funcionais para regiões mais internas, fazendo com que a molhabilidade tende ao estado de antes da modificação ${ }^{[21,33]}$. Ou seja, o valor do ângulo de contato tende ao de antes do tratamento, como pode ser visto na Figura 4. No caso da tratada por VUV, uma hipótese para o 
envelhecimento mais lento seria a reticulação da superfície, o que faria com que a mobilidade das cadeias seja reduzida ${ }^{[26]}$. Mesmo assim, alguns grupos funcionais migrariam para regiões mais internas $^{[21,33]}$.

Para a análise da modificação química, foram obtidos espectros ATR-FTIR das amostras envelhecidas por 14 e 28 dias, tanto da amostra tratada por plasma (Figura 5a), quanto por VUV (Figura 5b). Como se pode observar, a banda relativa aos grupos $[\mathrm{OH}]$ e $[\mathrm{CO}]$ da amostra tratada por plasma diminui com o tempo de envelhecimento, desaparecendo praticamente em 14 dias. Tal desaparecimento pode ser justificado pela hipótese apresentada por Yasuda et al. ${ }^{[33]}$ já citada anteriormente. No caso da amostra tratada por VUV, a banda relativa ao grupo $[\mathrm{OH}]$ só desaparece após 28 dias, enquanto que a banda relativa ao grupo [CO] mesmo após 28 dias ainda permanece. Resultados parecidos foram obtidos por outros autores ${ }^{[16,34]}$.

A Figura 6 mostra a representação 3D das imagens obtidas por microscopia de força atômica das amostras não tratada (PP), tratada por plasma por 20 minutos (P20), tratada por VUV por 20 minutos (V20) e tratadas por VUV e envelhecidas por 14 (14 dias) e 28 dias (28 dias). Antes do tratamento, a superfície é lisa, com poucos picos. Após 20 minutos de exposição ao plasma de $\mathrm{O}_{2}$ (P20), a superfície apresenta elevada rugosidade $(43 \mathrm{~nm})$. Para a amostra tratada por VUV por 20 minutos (V20), a superfície também apresenta certa rugosidade $(7,0 \mathrm{~nm})$, porém, bem menor que a tratada por plasma. Esta diferença na topografia evidencia que a mudança estrutural nos processos envolvendo plasma é maior que nos processos envolvendo apenas radiação. No tratamento por plasma, a energia transferida para a superfície pelas espécies ativas (principalmente íons) é maior que aquela transferida pelos fótons, presentes tanto no plasma (na região da descarga luminescente) como nos processos envolvendo radiação UV e VUV. O valor da energia dos fótons que atingem a superfície pode ser calculado pela relação de Einstein $(\mathrm{E}=\mathrm{hV})$. No caso da lâmpada utilizada neste trabalho, a energia dos fótons é cerca de 7,5 eV. Dado as condições de tratamento por plasma (pressão e potência da fonte) a energia dos íons que atingem a superfície é da ordem de $20 \mathrm{eV}$.

Com relação ao envelhecimento da amostra tratada por VUV, observa-se que a superfície tende a ficar lisa, apesar de ondulada. Ou seja, sua topografia tende ao da amostra não tratada. Com isso, o valor da rugosidade diminui com o tempo de envelhecimento, indicando que as cadeias mais externas da superfície se reorganizam ao longo do tempo. Tal tendência é suportada pela hipótese do rearranjamento das cadeias do polímero modificado citada acima.

\section{Conclusões}

Neste trabalho amostras de polipropileno foram tratadas por VUV (irradiadas em $\mathrm{N}_{2}$ e depois imersas em $\mathrm{O}_{2}$ ) e por plasma (descarga RF de oxigênio). As mudanças observadas para a molhabilidade das amostras tratadas por VUV ocorreram devido principalmente a uma mudança química da superfície uma vez que a rugosidade praticamente não é alterada. Por outro lado, as superfícies das amostras tratadas por plasma apresentaram tanto variações topográficas quanto químicas. No caso do envelhecimento, as amostras tratadas por plasma envelheceram mais rapidamente que as amostras tratadas por VUV, devido provavelmente a reorientações das cadeias de forma mais rápida. Além disto, uma provável reticulação da superfície faz com que o ângulo de contato aumente de forma mais lenta para a amostra tratada por VUV.

\section{Agradecimentos}

Os autores agradecem ao Prof. Luis Henrique Alves Candido (Lab. de Design e Seleção de Materiais - Dep. de Materiais - Escola de Engenharia/UFRGS) pela construção da câmara VUV. Também agradecemos a CAPES e ao CNPq pelo suporte financeiro.

\section{Referências Bibliográficas}

1. Köhler, L.; Scaglione, S.; Flori, D.; Riga, J. \& Caudano, R. - Nucl. Instr. Meth. B, 185, p.267 (2001).

2. Lee, J-W.; Son, S-M. \& Hong, S-I. - J. Food. Eng., 86, p.483 (2008).

3. Novak, I.; Pollak, V. \& Chodak, I. - Plasma Process. Polym., 3, p.355 (2006).

4. Yovcheva, T. A.; Avramova, I. A.; Mekishev, G. A. \& Marinova, T. S. - J. Electrostat., 65, p.667 (2007). http://dx.doi.org/10.1016/j. elstat.2007.05.002

5. Labay, C.; Canal, C. \& García-Celma, M. J. - Plasma Chem. Plasma Process., 30, p.885 (2010). http://dx.doi.org/10.1007/s11090-010-9255-2

6. Bullett, N. A.; Bullett, D. P.; Truica-Marasescu, F.-E.; Lerouge, S.; Mwaleb, F. \& Wertheimer, M. R. - App. Surf. Sci., 235, p.395 (2004). http://dx.doi.org/10.1016/j.apsusc.2004.02.058

7. Hozumi, A.; Shirahata, N.; Nakanishi, Y.; Asakura, S. \& Fuwa, A. - J. Vac. Sci. Technol. A, 22, p.1309, (2004). http://dx.doi. org/10.1116/1.1701867

8. Sarantopoulou, E.; Kova, J.; Kollia, Z.; Raptis, I.; Kobed, S. \& Cefalas, A. C. - Surf. Interface Anal., 40, p.400 (2008). http://dx.doi. org/10.1002/sia.2776

9. Truica-Marasescu, F-E. \& Wertheimer, M. R. - Macromol. Chem. Phys., 206, p.744 (2005). http://dx.doi.org/10.1002/macp.200400510

10. Truica-Marasescu, F.; Guimond, S.; Jedrzejowski, P. \& Wertheimer, M.R. - Nucl. Instr. Meth. B, 236, p.117 (2005). http://dx.doi. org/10.1016/j.nimb.2005.03.266

11. Wu, S-S.; Ran, Q-P. \& Shen, J. - J. Thermoplast. Compos. Mat., 23, p.149 (2010).

12. Wilken, R.; Holländer, A. \& Behnisch, J. - Plasmas Polym., 7, p.185 (2002). http://dx.doi.org/10.1023/A:1016247524912

13. Vasconcellos, A. S.; Stocker, C. H. F. \& Baumhardt Neto, R. - Polímeros, 7, p.44 (1997). http://dx.doi.org/10.1590/S010414281997000100008

14. Kwon, O-J.; Myung, S-W.; Lee, C-S. \& Choi, H-S. - J. Colloid. Interface. Sci., 295, p.409 (2006). PMid:16310799. http://dx.doi. org/10.1016/j.jcis.2005.11.007

15. Garcia, D.; Sanchez, L.; Fenollar, O.; Lopez, R. \& Balart, R. - J. Mater. Sci., 43, p.3466 (2008). http://dx.doi.org/10.1007/s10853-007-2322-2

16. Sorrentino, L.; Carrino, L. \& Napolitano, G. - Surf. Eng., 23, p.247 (2003). http://dx.doi.org/10.1179/174329407X215104

17. Pandiyaraj, K. N.; Selvarajan, V.; Deshmukh, R. R. \& Gao, C. Vacuum, 83, p.332 (2009).

18. Kauling, A. P.; Soares, G. V.; Figueroa, C. A.; Oliveira, R. V. B.; Baumvol, I. J. R.; Giacomelli, C. \& Miotti, L. - Mater. Sci. Eng. C Mater. Biol. Appl., 29, p.363 (2009).

19. Grundke, K. - "Characterization of Polymer Surfaces by Wetting and Electrokinetic Measurements - Contact Angle, Interfacial Tension, Zeta Potential", in: Polymer Surface and Interfaces, p.103-114, M. Stamm (ed.), Springer, Berlin (2008). http://dx.doi.org/10.1007/978-3-54073865-7_6

20. Chan, C-M.; Ko, T-M. \& Hiraoka, H. - Surf. Sci. Rep., 24, p.1 (1996). http://dx.doi.org/10.1016/0167-5729(96)80003-3

21. Strobel, M.; Jones, V.; Lyons, C. S.; Ulsh, M.; Kushner, M. J.; Dorai, R. \& Branch, M. C. - Plasmas Polym., 8, p.61 (2003). http://dx.doi. org/10.1023/A:1022817909276

22. Mirabedini, S. M.; Arabi, H.; Salem, A. \& Asiaban, S. - Progress in Organic Coatings, 60, p.105 (2007). http://dx.doi.org/10.1016/j. porgcoat.2007.07.007 
23. Kwon, O-J.; Tang, S.; Myung, S-W.; Lu, N. \& Choi, H-S. - Surf. Coat. Tech., 192, p.1 (2005). http://dx.doi.org/10.1016/j.surfcoat.2004.09.018

24. Truica-Marasescu, F.; Jedrzejowski, P. \& Wertheimer, M. R. Plasma Process. Polym., 1, p.153 (2004). http://dx.doi.org/10.1002/ ppap. 200400020

25. Pandiyaraj, K. N.; Selvarajan, V.; Deshmukh, R. R. \& Gao, C. App. Surf. Sci., 255, p.3965 (2009). http://dx.doi.org/10.1016/j. apsusc.2008.10.090

26. Truica-Marasescu, F.; Guimond, S.; Jedrzejowski, P. \& Wertheimer, M. R. - Nucl. Instr. Meth. B, 236, p.117 (2005). http://dx.doi. org/10.1016/j.nimb.2005.03.266

27. Wanke, C. H.; Feijó, J. L.; Barbosa, L. G.; Campo, L. F.; Oliveira, R. V. B. \& Horowitz, F. - Polym., 52, p.1797 (2011). http://dx.doi. org/10.1016/j.polymer.2011.01.064

28. Kim, Y-J.; Taniguchi, Y.; Murase, K.; Taguchi, Y. \& Sugimura, H. App. Surf. Sci., 255, p.3648 (2009). http://dx.doi.org/10.1016/j. apsusc.2008.10.009
29. Occhiello, E.; Morra, M.; Cinquina, P. \& Garbassi, F. - Polym., 33, p.3007 (1992). http://dx.doi.org/10.1016/0032-3861(92)90088-E

30. Arefi-Khonsari, F.; Kurdi, J.; Tatoulian, M. \& Amouroux, J. - Surf. Coat. Tech., 142-4, p.437 (2001).

31. Garbassi, F.; Morra, M.; Occhiello, E.; Barino, L. \& Scordamaglia, R. - Surf. Interface Anal., 14, p.585 (1989). http://dx.doi.org/10.1002/ sia.740141004

32. Kuzuya, M.; Noguchi, A.; Ito, H.; Kondo, S-I. \& Noda, N. J. - Polym. Sci. Polym. Chem. Ed., 29, p.1 (1991). http://dx.doi.org/10.1002/ pola.1991.080290102

33. Yasuda, H. \& Sharma, A. K. - J. Plasma Sci. Polym. Phy. Ed., 19, p.1285 (1981). http://dx.doi.org/10.1002/pol.1981.180190901

34. Boyd, R. D.; Kenwright, A. M.; Badyal, J. P. S. \& Briggs, D. Macromol., 30, p.5429 (1997). http://dx.doi.org/10.1021/ma960940x

Enviado: 06/05/11 Reenviado: 28/06/11 Aceito: $22 / 07 / 11$ 